\title{
EPIDEMIOLOGIA DA LEISHMANIOSE VISCERAL EM UM MUNICÍPIO DA BAHIA
}

\section{EPIDEMIOLOGY OF VISCERAL LEISHMANIOSIS IN A MUNICIPALITY OF BAHIA}

\begin{abstract}
Polyana Leal Silva, Tâmina de Lima Alves, Paloma Natal Teixeira, Josiane dos Santos Pereira, Milena Thaís Vilasboas Gomes, Marcela Andrade Rios
\end{abstract}

Universidade do Estado da Bahia - UNEB

\begin{abstract}
To describe the reported and confirmed cases of visceral leishmaniasis in a municipality in the interior of Bahia, from 2008 to 2012, regarding the sociodemographic aspects of the individuals and investigation of the cases. This is a descriptive and cross-sectional epidemiological study, carried out between April and August 2014 in the municipality of Guanambi-BA, using data from SINAN and the Department of Epidemiological Surveillance, referring to the data of notification and LV confirmation for the years 2008 to 2012. There were 111 notifications in the period, with 14 notifications related to the year 2008 (12.6\%), 26 (23.4\%) of 2009, 27 (24.3\%), of 2010, 22 (19.8\%) in 2011 and also 19.8\% in 2012. The male gender was the most frequent, in the age group under 10 years of age. Of the total cases reported, $73 \%$ were confirmed, especially by laboratory criteria. The mortality rates found were increasing in the years studied, reaching $13.6 \%$ in 2012. Therefore, it is emphasized the need for preventive measures, as well as the quality of diagnostic and treatment assistance, seeking to avoid new cases and the involvement of deaths and sequelae.
\end{abstract}

Key words: Endemic Diseases, Epidemiology, Leishmaniasis Visceral.

\section{Resumo}

Trata- se de um estudo descritivo e transversal, de cunho epidemiológico, realizado entre os meses de abril e agosto de 2014 no município de Guanambi- BA, utilizando de dados do SINAN e do Departamento de Vigilância Epidemiológica, referente aos dados de notificação e de confirmação deLV para os anos de 2008 a 2012, com o objetivo de descrever os casos notificados e aqueles confirmados de leishmaniose visceral, no período de 2008 a 2012, quanto aos aspectos sociodemográficos dos indivíduos e investigação dos casos. Foram encontradas 111 notificações no período, sendo 14 notificações relativas ao ano de 2008 (12,6\%), 26 (23,4\%) de 2009, 27 (24,3\%) de 2010, 22 (19,8\%) em 2011 e também $19,8 \%$ em 2012. O sexo masculino foi o mais frequente, na faixa etária menor de 10 anos de idade. Do total de casos notificados, $73 \%$ foram confirmados, especialmente pelo critério laboratorial. As taxas de letalidades encontradas foram crescentes nos anos estudados, chegando a 13,6\% em 2012. Portanto,enfatiza-se a necessidade de medidas preventivas, bem como a qualidade de assistência diagnóstica e de tratamento, buscando evitar novos casos e o acometimento de óbitos e sequelas.

Palavras chave: Doença Endêmica, Epidemiologia, Leishmaniose Visceral. 


\section{Introdução}

A Leishmaniose visceral (LV), popularmente conhecida como calazar, é considerada uma zoonose que acomete vísceras, como o fígado e o baço, podendo ocasionar o aumento de volume abdominal, assim como a perda da função dos mesmos12. É considerada como um problema de saúde pública no país, especialmente devido ao seu caráter endêmico em vários estados brasileiros.

A principal dinâmica de transmissão do parasita para os mamíferos se dá através da picada da fêmea infectada de L. longipalpis, que se encontra bem adaptada ao ambiente intra e peridomiciliar às diferentes temperaturas $\mathrm{e}$ alimentando-se em uma grande variedade de hospedeiros vertebrados, entre aves, homens e outros animais silvestres ou domésticos ${ }^{3}$.

A referida patologia possui caráter crônico infeccioso sistêmico, cujas manifestações clínicas são caracterizadas por febre irregular de intensidade média e de longa duração, acompanhado de anemia, leucopenia, trombocitopenia, hipergamaglobulinemia e hipoalbuminemia. Tais manifestações levam a pessoa acometida pela patologia a um estado de desnutrição e emagrecimento acentuado, podendo evoluir para óbito ${ }^{2}$. O diagnóstico baseia-se nos sinais e sintomas associados aos parâmetros epidemiológicos, assim como a sua confirmação se dá através de amostras biológicas do parasita em tecidos do paciente ${ }^{2}$.

Estima-se uma incidência anual no mundo de cerca de 500.000 casos, 59.000 óbitos e 200 milhões de pessoas sob o risco de adoecer ${ }^{4}$. No Brasil, a doença está presente nas cinco regiões, sendo um desafio para a saúde pública, especialmente para a região Nordeste por ser a mais pobre e possuir condições ambientais favoráveis à proliferação dos vetores responsáveis, apresentando mais de $90 \%$ dos casos registrados ${ }^{5}$.

Desde a década de 1970 observa- se o fenômeno da urbanização de forma crescente e acentuada, levando a mudanças no perfil epidemiológico comum da doença. Antes tida como uma doença rural, passou a afetar áreas urbanas, ocorrendo atualmente em grandes centros urbanizados, revelando assim o processo supracitado da patologia ${ }^{6}$.

Conforme dados disponibilizados pelo Departamento de Informática do SUS - Datasus ${ }^{7}$, a taxa de incidência de leishmaniose visceral, segundo a Unidade de Federação no período de 2012, mostrou valores mais elevados para o estado de Tocantins, seguido de Mato Grosso do Sul, Piauí, Maranhão e outros estados nordestinos.

Consequentemente, os focos endêmicos da doença, em sua maioria, estão localizados nas periferias e no âmbito rural, onde encontram-se condições de saneamento básico inadequado, depósitos de lixo, dentre outros locais ${ }^{5}$.

Nos últimos dez anos, apesar dos recursos de tratamento intensivo e das rotinas estabelecidas para o tratamento específico da LV, constatou-se aumento na letalidade da doença em diversas regiões do país. Um dos principais fatores que contribuem para o aumento dessa letalidade é o diagnóstico tardio, devendo-se priorizara capacitação dos profissionais das Unidades de Saúde da Família ${ }^{5}$.

Com base no exposto, o presente estudo, ao descrever os casos notificados e confirmados, revela sua importância ao demonstrar que, apesar dos esforços no sentido de combater a endemia, não tem sido o suficiente para erradicála, vez que a mesma acomete pessoas nas quais o sistema imunológico encontra-se deficiente, sendo agravado pela desnutrição, bem como as condições socioeconômicas em que vivem, fazendo-se necessária uma política para melhor atender esses indivíduos e consequentemente proporcionar uma melhora no quadro da incidência da leishmaniose visceral.

Por se tratar de uma doença de notificação compulsória ao Sistema de Notificação de Agravos (SINAN), a vigilância epidemiológica tem por objetivo informar e investigar os casos, reduzir as taxas de letalidade e grau de morbidade através do diagnóstico e tratamento precoce dos casos, bem como diminuir os riscos de transmissão mediante o controle da população de reservatórios e do agente transmissor.

Este estudo tem como objetivo descrever os casos notificados e àqueles confirmados de leishmaniose visceral em um município do interior da Bahia, no período de 2008 a 2012, quanto aos aspectos sociodemográficos dos indivíduos e investigação dos casos.

\section{Metodologia}

Trata-se de um estudo descritivo e transversal, de cunho epidemiológico, realizado entre os meses de abril e agosto de 2014, com base em dados sobre leishmaniose visceral referente aos indivíduos residentes em Guanambi, cidade localizada na região sudoeste 
do estado da Bahia, com população estimada pelo IBGE em 85.237 habitantes para o ano de 2014, e por ser considerado como um dos municípios que apresentam transmissão intensa de leishmaniose no estado ${ }^{8}$.

O período escolhido foi de 2008 a 2012 por corresponder aos anos com dados disponíveis até o momento na base de dados do Sistema de Informação de Agravos de Notificações (SINAN) e disponibilizados eletronicamente.

A coleta de dados ocorreu em dois momentos e utilizando duas fontes diferentes: dados disponibilizados pela Divisão de Vigilância Epidemiológica do município e referentes aos casos notificados e dados dos casos de LV confirmados oriundos do SINAN e acessados eletronicamente por meio do Departamento de Informática do SUS (Datasus).

Para descrever as características das notificações de leishmaniose visceral foram estudadas as variáveis sexo, faixa etária, local de notificação e mês de notificação. Foram estimados os coeficientes de incidência de LV para cada ano estudado por meio da divisão do número de casos confirmados pela população residente no município, multiplicado por 100.000 .
As variáveis critério de evolução e confirmação também foram estudadas para melhor descrever os casos confirmados.

Os dados foram organizados e analisados descritivamente com o auxílio do programa Microsoft ${ }^{\circledR}$ Office Excel 2010, com os cálculos de frequência absoluta e relativa, criação de tabelas e figuras.

\section{Resultados e Discussões}

Foram encontradas 111 notificações de Leishmaniose Visceral no período de janeiro de 2008 a dezembro de 2012, sendo 14 notificações $(12,6 \%)$ relativas ao ano de $2008,26(23,4 \%)$ de 2009, 27(24,3\%) de 2010, 22(19,8\%) em 2011 e também 19,8\% em 2012.

Conforme a Tabela 1, em relação aos casos notificados quanto ao sexo, pode-se observar que $64 \%$ dos indivíduos eram do sexo masculino e a faixa etária mais acometida foi em menores de 10 anos de idade $(43,2 \%)$. Para os casos confirmados manteve-se uma maior frequência em indivíduos do sexo masculino (64,2\%) e na mesma faixa etária $(48,1 \%)$.

Tabela 1 - Distribuição dos casos de Leishmaniose visceral notificados e confirmados segundo sexo e faixa etária, no município de Guanambi, nos anos de 2008 a 2012

\begin{tabular}{|c|c|c|c|c|}
\hline \multirow[t]{2}{*}{ Variáveis } & \multicolumn{2}{|c|}{$\begin{array}{c}\text { Casos } \\
\text { Notificados }\end{array}$} & \multicolumn{2}{|c|}{$\begin{array}{c}\text { Casos } \\
\text { Confirmados }\end{array}$} \\
\hline & $\mathbf{N}$ & $\%$ & $\mathbf{N}$ & $\%$ \\
\hline \multicolumn{5}{|l|}{ Sexo } \\
\hline Masculino & 71 & 64 & 52 & 64,2 \\
\hline Feminino & 40 & 36 & 29 & 35,8 \\
\hline \multicolumn{5}{|l|}{ Faixa Etária (Em Anos) } \\
\hline Menor De 10 & 48 & 43,2 & 39 & 48,1 \\
\hline 10 A 19 & 11 & 9,9 & 07 & 8,6 \\
\hline 20 A 39 & 26 & 23,4 & 17 & 21,0 \\
\hline 40 A 59 & 18 & 16,2 & 10 & 12,3 \\
\hline $60 \mathrm{E}+$ & 08 & 7,2 & 07 & 8,6 \\
\hline Total & 111 & 100 & 81 & 100 \\
\hline
\end{tabular}

Fonte: Vigilância Epidemiológica de Guanambi, BA; DATASUS, Ministério da Saúde, 2014.

Quando analisados os bairros com notificações de LV efetuadas pelo Departamento de Vigilância Epidemiológica do município, identificou-se que dos 93 bairros existentes no município, 32 (34,4\%) apresentaram notificações, sendo que as localidades com maior número de casos foram o Monte Pascoal com 14 notificações $(12,6 \%)$, Ipiranga com $12(10,8 \%)$, seguido do Vomitamel, Alvorada e Beija Flor com 7 casos (6,3\%) em cada um. Os bairros Paraíso, São Francisco, Alto Caiçara e a Zona Rural com 6 notificações $(5,4 \%)$, BNH e Santa Luzia com 4 $(3,6 \%)$, Centro, Amambaí e Lagoinha com 3 $(2,7 \%)$, no Loteamento Dr. Beneval Boa Sorte e Vasconcelos 02 casos (1,8\%) registrados, e mais 17 bairros com 1 caso notificado em cada $(0,9 \%)$. 


\section{Casos Confirmados}

Do total de casos notificados pela Vigilância Epidemiológica, 81 (73\%) obtiveram confirmação após a investigação.

$\mathrm{Na}$ Figura 1 observa-se a evolução na incidência de Leishmaniose Visceral no período de 2008 a 2012, revelando um aumento do número de casos no ano de 2010,21 (26,6\%) casos registrados, onde em 2008 eram 11 (13,9\%) casos, representando um aumento de $12,7 \%$ no período.

Figura 1- Evolução da incidência de leishmaniose visceral no Município de Guanambi-BA, nos anos de 2008 a 2012, por 100.000 habitantes.

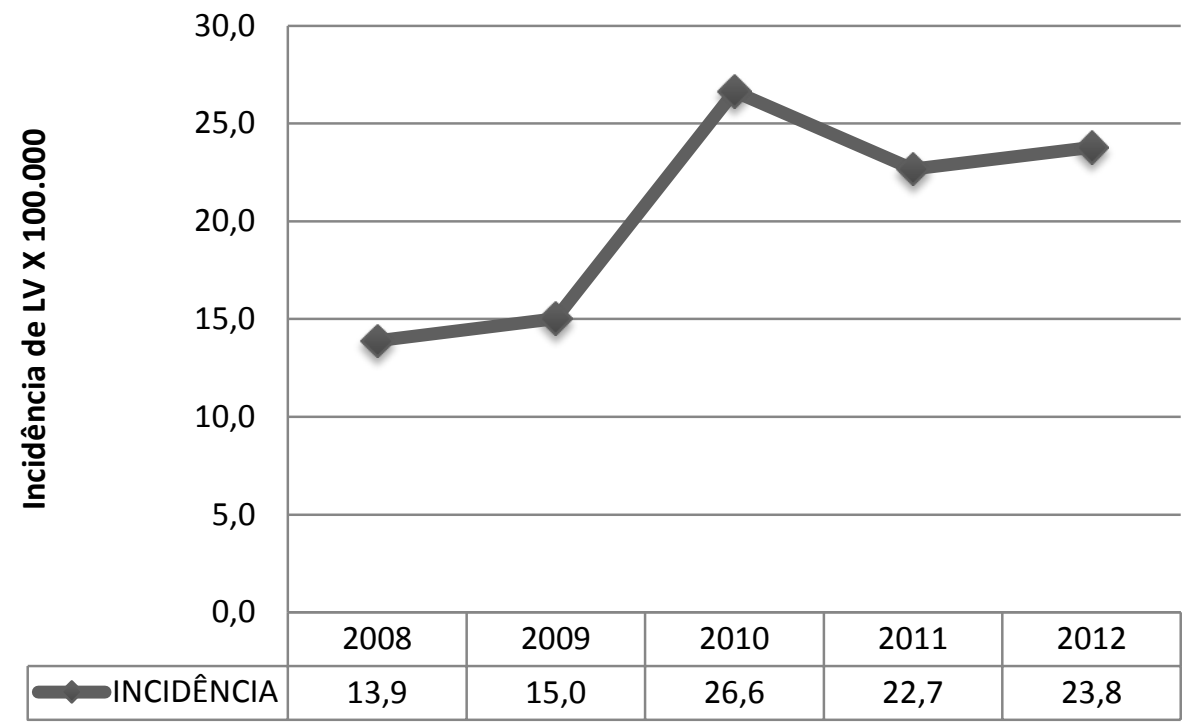

Fonte: DATASUS, Ministério da Saúde, 2014.

Conforme Figura 2, não foi encontrado um padrão nos meses de confirmação de casos de LV, havendo dispersão no período estudado. Contudo, observam-se meses com maiores frequências de casos confirmados de LV, a saber: janeiro $14(17,3 \%)$ casos, março $10(12,3 \%)$ casos e agosto $10(12,3 \%)$ casos registrados.

Figura 2- Distribuição dos casos confirmados de Leishmaniose Visceral, no município de Guanambi, segundo os meses e anos.

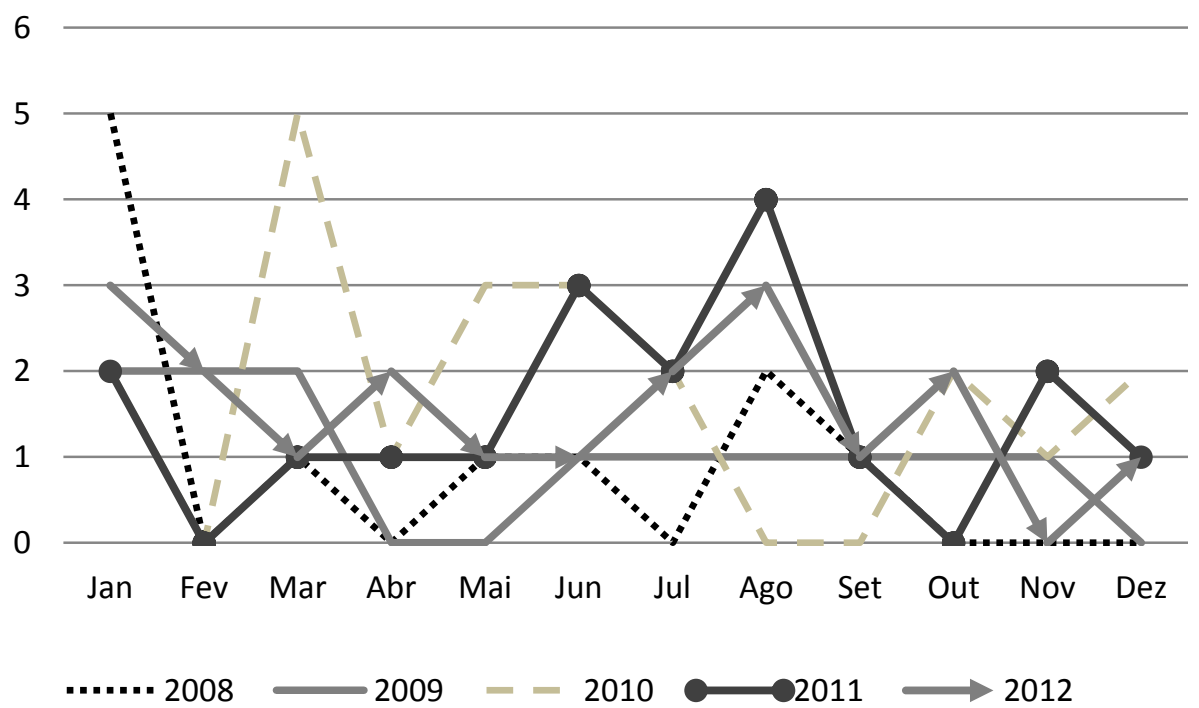


Segundo dados dos casos confirmados, pode-se observar quanto ao critério de confirmação da LV que o mais frequente foi a confirmação laboratorial, com 17 casos (21\%), seguido pelo epidemiológico com 12 casos $(14,8 \%)$ confirmados. Ressalta-se que foram encontradas informações para 29 casos $(35,8 \%)$ dos 81 apresentados.

Tabela 2- Distribuição dos casos confirmados de Leishmaniose Visceral, segundo a variável evolução dos casos em cada período.

Anos

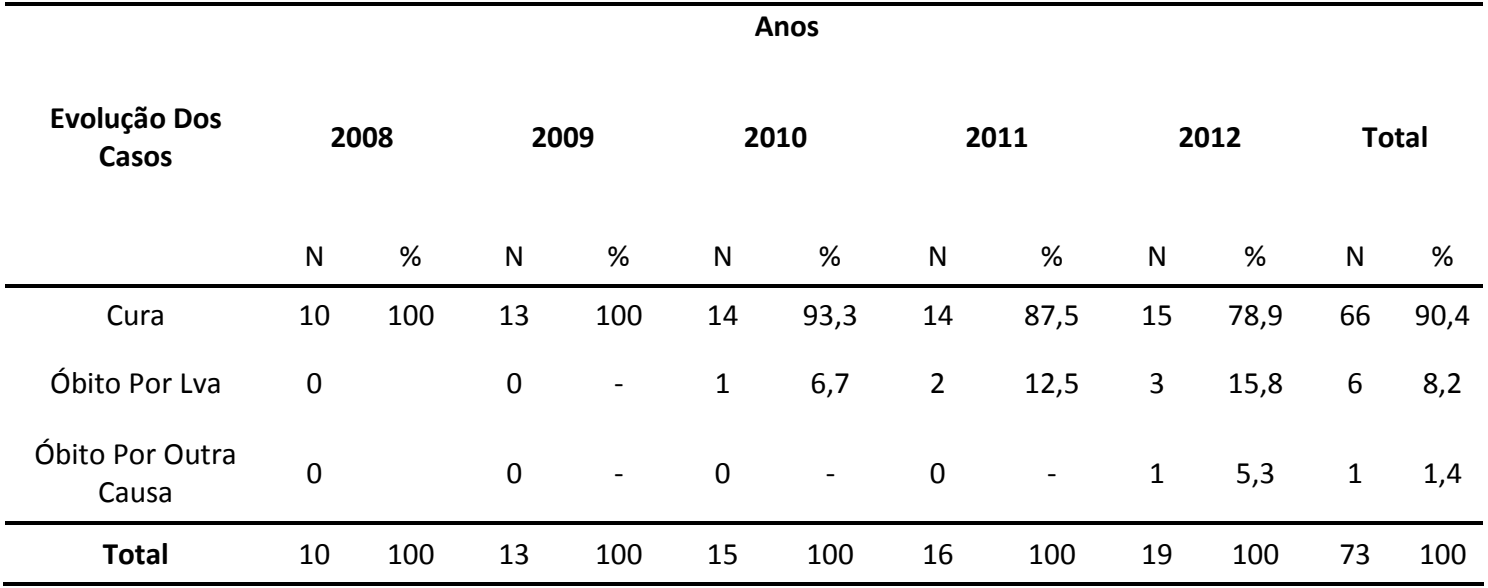

Fonte: Datasus, Ministério Da Saúde, 2014.

Quanto à variável evolução dos casos, para as categorias abandono de tratamento e transferência, não houve registros. Observa-se que 73 casos confirmados (90,1\%) obtiveram informações sobre o tipo de critério de evolução, apresentando maior frequência para curarem 66 casos(90,4\%). Foram registrados ainda 6 óbitos por LV, o que representa $8,2 \%$ do total de casos confirmados, sendo o ano de 2012 o que apresentou o maior número de óbitos, conforme visualizado na Tabela 2.

Os resultados dos cálculos de letalidade estimados apresentaram aumento para os anos observados, passando de nenhum caso em 2008 e 2009, para 3,7\% em 2010, 9,1\% em 2011 e 13,6\% em 2012.

Os achados do estudo mostram que houve no município de Guanambi uma incidência mais acentuada de LV em indivíduos do sexo masculino, com predominância em menores de 10 anos. No Brasil a LV clássica acomete pessoas de todas as idades, observando-se uma frequência acentuada em crianças menores de 10 anos $(54,4 \%)$, sendo $41 \%$ dos casos registrados em menores de 5 anos, o que pode ser justificado pela imaturidade imunológica celular, agravada pela desnutrição tão comum nas áreas endêmicas de abrangência ${ }^{5}$. O sexo masculino é proporcionalmente o mais afetado, como descrito em outros estudos disponíveis na literatura, e sugerem que homens estariam mais expostos ao vetor, possivelmente por exercerem suas atividades laborais mais próximas das fontes de infecção ${ }^{9,10}$.

Em concordância ${ }^{11}$, a LV tem sido mais frequente em crianças de até quatro anos, mas observa-se uma distribuição homogênea nas demais faixas etárias, em concordância com outros autores.

As principais consequências da LV no organismo humano estão relacionadas ao aumento de algumas vísceras como baço e fígado e, em consequência disso, apareceram diversos distúrbios como caquexia, anemia, edema e hipoalbuminemia, que vão ser mais prejudiciais em crianças, já que a mesma se encontra em processo de formação/desenvolvimento ${ }^{12,10}$.

Apesar dos esforços no controle de vetores e reservatórios, a doença encontra-se em rápida expansão, por isso representa uma ameaça para a população e preocupa as autoridades sanitárias. Embora tenham sido investidos recursos e estabelecidas rotinas para o tratamento específico da Leishmaniose Visceral, o país vem registrando um aumento na letalidade em diversas regiões ${ }^{13}$.

Analisando a série histórica do estudo, percebe-se que houve um aumento no número de casos notificados durante o período entre 2008 a 2010, decrescendo em 2011 e mantendo o número no ano seguinte. A elevação no número de casos notificados deve-se, possivelmente, dentre outros fatores, ao aumento e disponibilidade das notificações ao 
SINAN.

Em contrapartida, no ano de 2012 houve um aumento no número de casos confirmados, fato este que pode apresentar algumas possíveis explicações, tais como a melhoria dos critérios de notificação e confirmação e/ou mudanças ambientais que favoreceram a proliferação de vetores. No Brasil, as medidas de controle empregadas não têm apresentado efetividade suficiente para redução sustentável da prevalência, no entanto importantes avanços foram alcançados na redução da letalidade ${ }^{14}$.

A incidência da LV apresentou-se com um aumento no ano de 2010 , quando comparado ao ano de 2008, exibindo uma elevação de quase $100 \%$, diminuindo no ano de 2011 e tendendo para uma estabilidade no ano seguinte. Provavelmente essa elevação se deve ao aumento da disponibilidade das notificações ao SINAN, sendo que a queda nos anos seguintes pode ser explicada pelo fato de no ano de 2010 terem começado as campanhas contra a LV e os seus resultados aparecerem nos anos seguintes ${ }^{15}$.

Através dos dados de notificação obtidos pela Vigilância Epidemiológica da cidade de Guanambi pode-se perceber que os bairros com maior incidência de casos de LV foram Monte Pascoal, Ipiranga e Vomitamel, sendo estes bairros localizados na periferia da cidade, onde grande parte dos moradores é advinda de uma classe social baixa, podendo este fator ter influênciasobre $^{18} \circ$ fator da desnutrição, frequente em grupos populacionais de baixa condição socioeconômica e nos quais a doença é prevalente.

Quanto aos fatores sociais, ambientais e climáticos, estão diretamente envolvidos na evolução da doença ${ }^{17}$, já que cada localidade apresenta características próprias, o que torna difíceis as ações de combate à essa patologia. Com relação ao fator climático para incidência da LV, nos períodos chuvosos há maior densidade vetorial, resultando na multiplicação do mosquito, uma vez que o mesmo necessita da água para reprodução. Além disso, a imunidade é uma justificativa para a predileção pela infância, que deve-se ao fato de ser este grupo particularmente susceptível à infecção e à progressão para o estado mórbido por conta da imaturidade do sistema imunológico ${ }^{4}$. Quanto menor a resposta imunológica do paciente, menor será sua resposta terapêutica e maior será 0 aparecimento de novas infecções ${ }^{18}$.

Para a confirmação dos casos de LV notificados, o critério laboratorial foi o mais frequente. Para o Ministério da Saúde tal critério abrange no mínimo uma das seguintes situações: encontro do parasita nos exames parasitológicos, direto ou na cultura, reação de imunofluorescência reativa com titulo de 1:80 ou mais, desde que excluídos outros diagnósticos, testes imunocromatográficos, comumente conhecidos como teste rápido, que utilizam antígenos recombinantes ${ }^{19,2}$. O indivíduo tratado deve ser acompanhado durante seis meses ${ }^{5}$. Ao final desse período, se o estado do paciente permanecer estável, ele será considerado clinicamente curado.

Entre os casos confirmados observou-se que o critério epidemiológico foi o segundo mais utilizado, que abrange as situações de indivíduos clinicamente suspeitos, sem confirmação laboratorial, proveniente de área com transmissão de LV, mas com resposta favorável ao teste terapêutico ${ }^{19}$.

Com relação à evolução dos casos, neste estudo, a categoria cura apresentou maior frequência com $66(90,4 \%)$ casos entre os anos de 2008 a 2012 e não havendo registro de transferência e abandono do tratamento. Os critérios de cura são essencialmente clínicos e os primeiros sinais de resposta costumam ser inespecíficos como a melhora do apetite e do estado geral. O desaparecimento da febre acontece entre o segundo e o quinto dia de tratamento. $O$ ganho ponderal e a redução do volume do baço e do fígado podem ser verificados nas primeiras semanas, embora a regressão total possa levar alguns meses. Os parâmetros hematológicos melhoram a partir da segunda semana. $O$ processo de normalização das proteínas séricas ocorre de forma lenta, podendo durar meses. Com evolução clinica favorável, o controle parasitológico é dispensável 18,19

É relevante considerar que uma parcela dos indivíduos com LV foram a óbito, representando uma letalidade crescente e alta nos anos de 2011 e 2012, quando comparado ao estimado pelo Ministério da Saúde ${ }^{19}$. Segundo dados deste órgão, a letalidade por LV aumentou de 3,4\% em 1994 para 5,7\% em 2009, o que representou incremento de $67,6 \%{ }^{19}$. Nesse contexto, salientase a importância das medidas de controle e prevenção, bem como de diagnóstico e tratamento precoce.

Vale ressaltar que foi instituída a lei federal 12.604/ 12 que cria a Semana Nacional de Controle e Combate à Leishmaniose e que tem entre seus objetivos estimular ações educativas e preventivas, bem como promover debates e 
outros eventos sobre as políticas públicas de vigilância e controle da leishmaniose, apoiar as atividades de prevenção e combate à leishmaniose, organizadas e desenvolvidas pela sociedade civil ${ }^{4}$.

\section{Conclusões}

Pode-se perceber que a LV acomete com maior frequência crianças menores de 10 anos bem como revelar que o sexo masculino é o mais afetado e que os bairros onde há mais registros de casos são aqueles que possuem condições socioeconômicas desfavoráveis. Observa-se ainda que o critério de confirmação mais frequente foi o laboratorial, na mesma proporção em que foi bem negligenciado, uma vez que apenas $35,8 \%$ dos casos receberam o critério de confirmação.Quanto à evolução dos casos, a categoria cura foi a que apresentou maior parcela de registros.

Em decorrência das mudanças constantes na dinâmica de transmissão da patologia, sugerese realizar campanhas mais enfáticas no combate ao vetor, bem como desenvolver novas Politicas Públicas que visem à redução dos problemas socioeconômicas da população mais afetada. Levar à população o conhecimento sobre a patologia, especialmente contando com o apoio das Unidades de Saúde da Família, poderá ser uma ação efetiva para a prevenção e controle da doença, segundo o que preconiza a lei 12.604/12.

Portanto, verifica-se que a LV apresentou alta letalidade, necessitando de atenção especial, principalmente no que diz respeito às medidas de prevenção, controle, diagnóstico e tratamento.

\section{Referências}

1. WSPA- Sociedade Mundial de Proteção Animal. Leishmaniose visceral canina.

2. Um manual para clínico veterinário. Rio de Janeiro; 2011. p.3-9. [Citado 2016nov4]. Disponível em: http://pt.slideshare.net/layaneholanda/manualleishmaniose

3. Neves DP. Parasitologia Humana. São Paulo: Atheneu; 2011.

4. Alvar J, Vélez DI, Bern C, Herrero $M$, Desjeux P, Cano J,et al. Leishmaniasis Control Team. Leishmaniasis Worldwide and Global Estimates of Its Incidence.PloS One. 2012;7(5): 35671.
5. WHO. World Health Organization. Controlof the leishmaniases: Report of a meeatinf of the WHO Expert Coomitee on the Control of Leishmaniases, Geneva, 22-26 march 2010. Who Tech. Rep. Ser. Geneva; 2010.p.1-202. [Citado 2016 nov 10]. Disponível em: http://apps.who.int/iris/bitstream/10665/44412/ 1/WHO_TRS_949_eng.pdf

6. Ministério da Saúde, Secretaria de Vigilância em Saúde. Departamento de Vigilância Epidemiológica. Manual de vigilância e controle da leishmaniose visceral. 2014; 4(1): 1.[Citado 2016 nov 17]. Disponível em: http://bvsms.saude.gov.br/bvs/publicacoes/man ual_vigilancia_controle_leishmaniose_visceral_1 edicao.pdf

7. Lopes EGP, Magalhães DF, Silva JA, Haddad JPA, Moreira EC. Distribuição temporal e espacial da leishmaniose visceral em humanos e cães em Belo Horizonte- MG, 1993 a 2007. Arq. Bras. Med. Vet. Zootec. 2010; 62(5): 10621071.[Citado 2016 nov 12]. Disponível em: http://www.scielo.br/scielo.php?script=sci_artte xt\&pid=S0102-09352010000500007

8. Brasil. Departamento de Informática do Sistema Único de Saúde- DATASUS. [Citado 2016 nov 12]. Disponível em: http://tabnet.datasus.gov.br/cgi/tabcgi.exe?idb2 012/d0205.def

9. Sesab. Secretaria de Saúde do Estado da Bahia. Diretoria de vigilância epidemiológica da SESAB. Situação da Leishmaniose visceral (LV). Estado da Bahia, 2013. [Citado 2016 nov 19]. Disponível em: http://www.suvisa.ba.gov.br/sites/default/files/b oletim\%20de\%20leishmaniose\%20visceral 2013. pub\%201-6 0.pdf. Acesso em 26 jan 2017.

10. Alvarenga DG, Escalda PMF, Costa ASV, MonrealMTFD. Leishmaniose visceral: estudo retrospectivo de fatores associados à letalidade. Rev. Soc. Bras. Med. Trop. 2010; 43(2): 194-97. [Citado 2016 nov 20]. Disponível em: http://www.scielo.br/pdf/rsbmt/v43n2/17.pdf

11. Oliveira LS, Neto RVD,Braga PET. Perfil Epidemiológico dos Casos de Leishmaniose Visceral em Sobral, Ceará no período de 2001 a 2010. SANARE, Sobral. 2013; 12(1): 13-19.[Citado 2016 nov 20]. Disponível em: https://www.google.com.br/url?sa=t\&rct=j\&q=\& esrc $=s \&$ source $=$ web $\& c d=6 \& c a d=r j a \&$ uact $=8 \&$ ved =0ahUKEwid17uLz6nSAhUKjpAKHTQQAvkQFgg8 MAU\&url=https\%3A\%2F\%2Fsanare.emnuvens.co m.br\%2Fsanare\%2Farticle\%2Fdownload\%2F323 \%2F258\&usg=AFQjCNGar6w6MsmiOKdTszYRL_P 
xAPMmsA\&sig2=IGYv9zuk8ii4PzTvEvC2ww\&bvm $=$ bv. $148073327, \mathrm{~d} . \mathrm{Y} 2 \mathrm{I}$

12. Góes MAO, JeraldoVLS, Oliveira AS. Urbanização da leishmaniose visceral: aspectos clínicos e epidemiológicos em Aracaju, Sergipe, Brasil. RevBrasMedFam Comunidade. 2014;9(31): 119-26. [Citado 2017 jan 25]. Disponível em: http://dx.doi.org/10.5712/rbmfc9(31)685.

13. Barbosa, IR. Epidemiologia da Leishmaniose Visceral no estado do Rio Grande do Norte, Brasil. Revista de Epidemiologia e Controle de Infecção.2013; 3(1): 17-21. [Citado 2017 jan 25]. Disponível em: https://online.unisc.br/seer/index.php/epidemiol ogia/article/view/3148

14. Leite Al, Araújo LB. Leishmaniose Visceral: aspectos epidemiológicos relacionados aos óbitos em Mossoró-RN. RevPatol Trop.2013; 42 (3): 301-8. [Citado 2017 jan 26]. Disponível em:

http://www.revistas.ufg.br/index.php/iptsp/artic le/view/26928.

15. Sesab. Secretaria de Saúde do Estado da Bahia. Núcleo de Vigilância Epidemiológica. Informe Epidemiológico Leishmaniose Visceral. Estado do Ceará. 2016. [Citado 2017 jan 26]. Disponível em: http://www.saude.ce.gov.br/index.php/boletins

16. Resumo Contratual. Estado da Bahia.Diário Oficial de Guanambi (Fev 6, 2010). [Citado 2017 jan 26].

Disponível

em: http://www.guanambi.ba.gov.br/pdf/120748201 026011.pdf.

17. Silvana SE, Gaioso IAC. Leishmaniose Visceral no Estado do Pará. Artigo Original. Belém, Pará, Brasil. 2013. [Citado 2017 jan 27]. Disponível em: http://files.bvs.br/upload/S/01015907/2013/v27n2/a3671.pdf.

18. Borges LFNM, Lopes EGP, Freitas ACPD, Silva MX, Haddad JPA, Silva JAD, et al.Prevalência e distribuição espacial da leishmaniose visceral em cães do município de Juatuba, Minas Gerais, Brasil. Cienc. Rural. 2014; 44(2): 352-57. [Citado 2017 jan 27]. Disponível em: http://www.scielo.br/scielo.php?script=sci_artte xt\&pid=S0103-84782014000200025

19. Barata, RA. Controle da leishmaniose visceral no município de Porteirinha, estado de Minas Gerais, no período de 1998 a 2003.Rev. Soc. Bras. Med. Trop. 2011; 44(3): 386-88. [Citado 2017 jan 27]. Disponível em:http://www.scielo.br/pdf/rsbmt/v44n3/v44n 3a25.pdf
20. Ministério da Saúde. Leishmaniose visceral: recomendações clínicas para redução da letalidade. 2015.[Citado 2017 jan 27]. Disponível em:

http://bvsms.saude.gov.br/bvs/publicacoes/man ual recomendacoes redução letalidade leishm ania.pdf

\section{Endereço para Correspondência}

Universidade do Estado da Bahia - UNEB

e-mail: poly_leal@hotmail.com

Recebido em 02/03/2017

Aprovado em 14/07/2017

Publicado em 21/08/2017 